\title{
Stromal PTEN inhibits the expansion of mammary epithelial stem cells through Jagged-1
}

\author{
GM Sizemore ${ }^{\# 1,2,{ }^{*}, \text { S Balakrishnan }}{ }^{\# 1,2}$, AM Hammer $^{1,2}$, KA Thies $^{1,2}$, AJ Trimboli ${ }^{1,2}$, JA \\ Wallace $^{1}$, ST Sizemore ${ }^{1,3}$, RD Kladney ${ }^{1,2}$, SA Woelke ${ }^{1}$, L Yu$^{4}$, SA Fernandez ${ }^{4}$, A \\ Chakravarti $^{3}$, G Leone ${ }^{1,2,5}$, and MC Ostrowski ${ }^{1,2,{ }^{*}}$ \\ ${ }^{1}$ The Comprehensive Cancer Center, The Ohio State University, Columbus, Ohio 43210, USA \\ 2Department of Cancer Biology \& Genetics, The Ohio State University, Columbus, Ohio 43210, \\ USA \\ ${ }^{3}$ Department of Radiation Oncology, The Ohio State University, Columbus, Ohio 43210, USA \\ ${ }^{4}$ Department of Biomedical Informatics' Center for Biostatistics, The Ohio State University, \\ Columbus, Ohio 43210, USA \\ ${ }^{5}$ Department of Molecular Genetics, The Ohio State University, Columbus, Ohio 43210, USA \\ \# These authors contributed equally to this work.
}

\section{Abstract}

Fibroblasts within the mammary tumor microenvironment are active participants in carcinogenesis mediating both tumor initiation and progression. Our group has previously demonstrated that genetic loss of PTEN in mammary fibroblasts induces an oncogenic secretome that remodels the extracellular milieu accelerating ErbB2-driven mammary tumor progression. While these prior studies highlighted a tumor suppressive role for stromal PTEN, how the adjacent normal epithelium transforms in response to PTEN loss was not previously addressed. To identify these early events, we have evaluated both phenotypic and genetic changes within the pre-neoplastic mammary epithelium of mice with and without stromal PTEN expression. We report that fibroblast-specific PTEN deletion greatly restricts mammary ductal elongation and induces aberrant alveolar side-branching. These mice concomitantly exhibit an expansion of the mammary epithelial stem cell (MaSC) enriched basal/myoepithelial population and an increase in in vitro stem cell activity. Further analysis revealed that NOTCH signaling, specifically through NOTCH3, is diminished in these cells. Mechanistically, JAGGED-1, a transmembrane ligand for the NOTCH receptor, is downregulated in the PTEN-null fibroblasts leading to a loss in the paracrine activation of NOTCH signaling from the surrounding stroma. Reintroduction of JAGGED-1 expression

Users may view, print, copy, and download text and data-mine the content in such documents, for the purposes of academic research, subject always to the full Conditions of use:http://www.nature.com/authors/editorial_policies/license.html\#terms

*Correspondence: Gina M. Sizemore, Ph.D., Department of Cancer Biology \& Genetics, The Comprehensive Cancer Center, Ohio State University, 570A Biomedical Research Tower, 460 W. $12^{\text {th }}$ Avenue, Columbus, OH 43210, gina.sizemore@ osumc.edu OR Michael C. Ostrowski, Ph.D., Department of Cancer Biology \& Genetics, The Comprehensive Cancer Center, Ohio State University, 598 Biomedical Research Tower, 460 W. $12^{\text {th }}$ Avenue, Columbus, OH 43210, michael.ostrowski@ osumc.edu.

CONFLICT OF INTEREST

The authors declare no conflicts of interest.

Supplementary information accompanies the paper on the Oncogene website 
within the PTEN-null fibroblasts was sufficient to abrogate the observed increase in colony forming activity implying a direct role for stromal JAGGED-1 in regulation of mammary stem cell properties. Importantly, breast cancer patients whose tumors express both low stromal JAG1 and low stromal PTEN exhibit a shorter time to recurrence than those whose tumors express low levels of either alone suggesting similar stromal signaling in advanced disease. Combined, these results unveil a novel stromal PTEN-to-JAGGED-1 axis in maintaining the mammary epithelial stem cell niche, and subsequently inhibiting breast cancer initiation and disease progression.

\section{Keywords}

Breast cancer; HER2/ErbB2; PTEN; Fibroblast; Mammary stem cells; Jagged-1; NOTCH3

\section{INTRODUCTION}

The tumor microenvironment (TME) plays an indispensable role in promoting mammary tumor initiation, progression and metastasis. ${ }^{1}$ Fibroblasts maintain tissue homeostasis throughout development by regulating a plethora of processes including extracellular matrix (ECM) deposition, growth factors and chemokine secretion, inflammation and wound healing. ${ }^{2,3}$ In breast cancer, cancer associated fibroblasts (CAFs) are aberrantly activated by largely unidentified mechanisms to engender an environment that favors tumor growth. ${ }^{4,5}$ Despite comprehensive evidence supporting CAFs in the TME, little is known about the pathways within these cells that fuel tumor growth. Our group demonstrated that genetic ablation of the tumor suppressor Pten within the mammary stroma greatly accelerates HER2/Neu (MMTV-ErbB2) driven mammary tumorigenesis. ${ }^{6,7}$ These previous studies were the first to demonstrate that malignant transformation of tumor epithelium can depend on fibroblast-specific signaling. Importantly, decreased stromal PTEN was observed in a subset of breast cancer patients and inversely correlated with stromal pro-oncogenic activated AKT1. ${ }^{7}$ Although these studies established a tumor suppressive role for PTEN within the mammary stroma, the mechanisms by which pre-neoplastic epithelia are reprogrammed in response to altered signaling surrounding fibroblasts have not been elucidated

Mammary gland morphogenesis occurs predominantly postnatally whereby epithelial stem/ progenitor cells generate mammary ducts during puberty. ${ }^{8-13}$ Secretory alveoli then arise throughout estrus cycling, and become fully differentiated lobulo-alveoli during pregnancy and lactation. ${ }^{14}$ This process is highly dynamic and closely mirrors tumorigenesis in that the mammary epithelium proliferates, invades into the fat pad and stimulates angiogenesis in response to surrounding stromal cells, including fibroblasts. ${ }^{15}$ Thus, aberrant signaling between stroma and epithelia are likely to cause oncogenic disruptions within specific epithelial subpopulations. In this report, we show that genetic loss of stromal Pten elicits non-cell autonomous changes within associated pre-neoplastic epithelium resulting in stunted mammary ductal elongation and aberrant alveolar side-branching. This phenotype is paralleled by an expansion of the mammary stem cell (MaSC) enriched population. We further demonstrate that ablation of stromal PTEN decreases NOTCH signaling in the associated MaSC-enriched population, and NOTCH3, specifically, is decreased in the basal/ 
myoepithelium upon stromal PTEN deletion. Mechanistically, PTEN-null fibroblasts exhibit significantly decreased JAGGED-1, one of the ligands for the NOTCH receptor, resulting in diminished JAGGED-1/NOTCH3 paracrine signaling, and a subsequent increase in mammary stem cell activity. Importantly, low stromal PTEN and JAG1 correlates with shorter time to recurrence in breast cancer patients implying similar signaling in the TME.

\section{RESULTS}

\section{Loss of stromal PTEN restricts mammary ductal elongation and promotes aberrant alveolar side-branching}

Loss of stromal PTEN accelerates $M M T V$-ErbB2-driven mammary tumor growth, which occurs at $\sim 16-20 \mathrm{wks}$ of age. ${ }^{7}$ Since mammary gland development like tumorigenesis is regulated by the stromal microenvironment, we evaluated early changes in the adjacent epithelium associated with PTEN loss. ${ }^{9,} 15$ To this end, we compared pre-neoplastic mammary glands of post-pubertal virgin mice (8-9wks of age) in four genetic groups: Pten $^{f l / f l}$ (wild-type epithelium; wild-type stroma), Fsp-cre; Pten ${ }^{f l / f l}$ (wild-type epithelium; PTEN-null stroma), MMTV-ErbB2;Pten ${ }^{f l / f l}$ (ErbB2 epithelium; wild-type stroma) and MMTV-ErbB2;Fsp-cre;Pten fl/fl (ErbB2 epithelium; PTEN-null stroma). Importantly, ablation of stromal PTEN greatly impaired epithelial ductal elongation (Figure 1A,B) and increased alveolar side-branching in the presence or absence of ErbB2 (Figure 1A,C).

To explore how stromal PTEN alters the mammary epithelium, we analyzed gene expression between gravity prepped epithelium from wild-type versus stromal PTEN-null mice at $8 \mathrm{wks}$ of age previously published by our group. ${ }^{16}$ Unsupervised gene set enrichment analysis (GSEA) querying all the $\mathrm{C} 2$ curated genesets within the Molecular Signatures Database (MSigDB), indicated enrichment of an adipose stem cell gene set ${ }^{17}$ when comparing ${ }^{F}$ spcre; Pten $^{\text {fllfl }}$ to Pten ${ }^{f l / f l}$-associated epithelium (Supplementary Figure 1A,B). We confirmed this finding using mammary stem cell (MaSC) specific gene lists also available on MSigDB: genes known to be downregulated in MaSCs are de-enriched in Fsp-cre;Pten ${ }^{f l / f l}$ epithelium and genes upregulated in MaSCs are enriched in Fsp-cre;Pten ${ }^{f l / f l}$ epithelium

(Supplementary Figure 1C). ${ }^{18}$ These combined results indicate that ablation of fibroblast PTEN elicits genomic changes within adjacent pre-neoplastic epithelial subpopulations.

\section{Loss of stromal Pten expands the MaSC-enriched population}

MaSCs exist within the basal/myoepithelium to coordinate ductal expansion and are strictly regulated by stromal cells and ECM components. ${ }^{13,19-22}$ To determine if loss of fibroblast PTEN alters the mammary epithelial populations, we used well-described fluorescence activated cell sorting (FACS) markers to evaluate 8-9wk old control and experimental mice. ${ }^{23}$ First, we confirmed our technique using wild-type FVB/N animals with appropriate isotype controls (Supplementary Figure 2A,B). The purity of isolated $\mathrm{Lin}^{-} \mathrm{CD} 24^{+} \mathrm{CD} 29^{\mathrm{Hi}}$ MaSC-enriched basal/myoepithelium, $\mathrm{Lin}^{-} \mathrm{CD} 24^{+} \mathrm{CD} 29{ }^{\mathrm{Lo}} \mathrm{CD} 61^{+}$luminal progenitors, and $\mathrm{Lin}^{-} \mathrm{CD} 24^{+} \mathrm{CD} 2{ }^{\mathrm{Lo}}{ }^{\mathrm{CD}} 61^{-}$mature luminal epithelial cells was confirmed by quantitative real-time PCR (qRT-PCR) using Trp63, cKit, and Esr1 as established MaSC, luminal progenitor and mature luminal markers, respectively (Supplementary Figure 2C). 
FACS analyses of $P$ ten $^{f l / f l}$ and $F s p$-cre; $P$ ten ${ }^{f l / f l}$ mice revealed that mammary glands with PTEN-null stroma exhibited a significant expansion of the MaSC-enriched basal/ myoepithelial population (Figure 2A). Similarly, the MaSC-enriched pool was expanded in ErbB2;Fsp-cre;Pten ${ }^{f l f f l}$ mice (Figure 2C). In contrast, no significant changes were observed in the $\mathrm{Lin}^{-} \mathrm{CD} 24^{+} \mathrm{CD} 29^{\mathrm{Lo}}$ total luminal populations in the absence of stromal PTEN with or without ErbB2 (Figure 2A,C; right graphs). Moreover, further sorting the total luminal populations with CD61 revealed no significant changes in either the Lin$\mathrm{CD} 24^{+} \mathrm{CD} 29^{\mathrm{Lo}} \mathrm{CD} 61^{-}$mature luminal or $\mathrm{Lin}^{-} \mathrm{CD} 24^{+} \mathrm{CD} 29^{\mathrm{Lo}} \mathrm{CD} 61^{+}$luminal progenitor populations (Figure 2B,D). To extend this observation of increased MaSC-enriched cells, we assessed in vitro differentiation and stem cell activity. MaSC-enriched cells from ErbB2+ stromal PTEN-null mammary glands generated significantly more 3D organoids compared to control (Figure 3A), and these organoids exhibited increased dual cytokeratin-5/ cytokeratin-8 positivity demonstrating increased stem-like activity (Figure 3B). In corroboration, MaSC-enriched cells from fibroblast PTEN-null mice have significantly enhanced colony forming ability in vitro (Figure 3C), and demonstrate an enhanced ability to form mammospheres under non-adherent culture conditions (Figure 3D). Importantly, no loss of PTEN was detected in MaSC-enriched cells by indirect immunofluorescent staining (Supplementary Figure 3A,B).

\section{MaSC-enriched cells within a stromal PTEN-null background have decreased NOTCH3}

To assess the stromal PTEN-dependent changes within the adjacent MaSCs, we isolated MaSC-enriched populations from both ErbB2; Pten ${ }^{f l / f l}$ and ErbB2; Fsp-cre; Pten ${ }^{f l / f l}$ mice at 8 wks of age and performed genome wide expression profiling (Supplementary Figure 4A). Unsupervised evaluation by GSEA querying the C2 MSigDB revealed significant deenrichment of the NOTCH pathway in the MaSC-enriched cells from stromal PTEN-null glands (Figure 4A; Supplementary Figure 4B). Confirmatory qRT-PCR revealed that Notch3, which had the highest enrichment signal of the NOTCH receptors (Supplementary Figure 4B), was downregulated in epithelium from ErbB2; Fsp-cre; Pten $^{f l / f l}$ mice (Figure 4B). To verify Notch 3 specifically drives the signature, the remaining family members (Notch1, Notch2, Notch4) were concluded as unchanged (Supplementary Figure 4C). Lastly, we confirmed the NOTCH target gene Hes 1 was also downregulated supporting diminished NOTCH signaling (Figure 4C; Supplementary Figure 4B).

To confirm diminished NOTCH3 protein, we performed immunohistochemistry (IHC) staining in 8-9wk mammary glands. Discontinuous NOTCH3 staining was observed in the basal/myoepithelium of stromal PTEN-null mice with or without ErbB2 (Supplementary

Figure 5A). NOTCH3 staining was also observed in the luminal lineage within all the glands, albeit at lower levels, and so to accurately quantify NOTCH3 exclusively in the MaSC-containing basal/myoepithelium, we carried out dual-immunofluorescence for NOTCH3 and alpha-smooth muscle actin (a-SMA), a marker for the basal/myoepithelium (Figure 4D,E). We measured the percentage of NOTCH3 positive area with respect to aSMA positive area and found that NOTCH3 was significantly downregulated in the a-SMA positive MaSC-enriched basal/myoepithelium in stromal PTEN-null mammary glands (Figure 4B,D). Notably, Notch3 mRNA was unchanged in both the luminal progenitor and 
mature luminal populations (Supplementary Figure 5B) supporting a role for NOTCH3 specifically within the MaSC-enriched cells.

\section{PTEN-null fibroblasts promote increased stem cell activity through decreased JAGGED-1}

Given that NOTCH signaling is known to restrict MaSC activity in vivo ${ }^{24}$, we hypothesized that Fsp-cre mediated ablation of stromal Pten decreases NOTCH signaling in associated MaSC basal/myoepithelial cells in a non-cell autonomous, paracrine manner. NOTCH3 activation is mediated by JAGGED and DLL ligands. Since these ligands are single-pass transmembrane proteins requiring direct cell-cell contact for ligand mediated activation of Notch signaling 25,26 , we postulated that changes in NOTCH3 in the basal/myoepithelium could be due to decreased ligand expression in adjacent stromal fibroblasts and subsequent autoregulation. To test this possibility, we isolated primary mouse mammary fibroblasts (MMFs) from 8wk old Pten ${ }^{f l / f l}$ and Fsp-cre;Pten ${ }^{f l / f l}$ animals and evaluated Jag1, Jag2, DII1, DII3 and DII4 mRNA. Importantly, we observed significantly lower Jag1 mRNA expression in PTEN-null MMFs when compared to wild-type MMFs (Figure 5A; Supplementary Figure 6A). Jag2, DII1 and DII4 mRNA levels were unchanged, and DII3 was undetectable by qRT-PCR (Supplementary Figure 6A). Combined, these results imply that low JAGGED-1 is specifically responsible for the observed phenotype. To further validate these findings, we evaluated Jag1 mRNA in a pair of immortalized wild-type and PTEN-null MMF cells ${ }^{16}$, observing almost complete loss of JAGGED-1 mRNA and protein (Figure 5B,C; Supplementary Figure 6B). We further validated these results in vivo through dual immunofluorescence for JAGGED-1 and a-SMA in mammary gland sections from 8-9wk old Pten ${ }^{f l / f l}$, Fsp-cre;Pten ${ }^{f l / f l}$,ErbB2;Pten ${ }^{f l / f l}$, and ErbB2;Fsp-cre;Pten ${ }^{f l / f l}$ mice. A decrease in stromal JAGGED-1 is observed in glands lacking stromal PTEN (Figure 5D,E) suggesting fibroblast PTEN mediates stromal-to-epithelial cross-talk through stromal JAGGED-1.

To test whether cross talk from the PTEN-null MMFs alters MaSC activity directly, we isolated Lin-CD $24^{+} \mathrm{CD} 29^{+}$mammary epithelial cells from $E r b B 2$ mice and cultured these cells on growth arrested wild-type or PTEN-null immortalized MMFs. PTEN-null MMFs greatly enhanced the colony forming ability of purified $\operatorname{Er} b B 2$ epithelium confirming a noncell autonomous role for these fibroblasts in the stem-like capacity of adjacent epithelium (Figure 6A). To test whether JAGGED-1 downregulation in the PTEN-null MMFs is directly responsible for the increased stem cell activity, we ectopically expressed JAGGED-1 in the PTEN-null MMFs (Supplementary Figure 6C) and found that JAGGED-1 overexpression abrogated the increased epithelial colony forming ability (Figure 6B). Importantly, NOTCH inhibition via a $\gamma$-Secretase inhibitor (GSI-XXI) increases the colony forming capacity of $E r b B 2$ epithelium on wild-type MMFs further supporting a role for low JAGGED-1/NOTCH signaling in associated epithelial stem cell activity (Figure 6C).

\section{Low expression of stromal JAG1 and stromal PTEN correlates with shorter time to recurrence in breast cancer patients}

To test the relevance of stromal PTEN-mediated JAGGED-1 signaling in human breast cancer, we evaluated publicly available gene expression data of 53 laser-captured primary breast tumor samples for stromal $J A G 1$ and PTEN expression. ${ }^{27}$ Patients were segregated into quartiles depending on stromal expression of $J A G 1$, and survival was compared between 
patients with high (top 75\%) and low (bottom 25\%) JAG1. Low stromal $J A G 1$ (Figure 7A), and similarly, low stromal PTEN(Figure 7B) correlates with shorter time to recurrence in patients. Importantly, patients with a combination of low stromal PTEN and JAG1 exhibited the shortest time to recurrence when compared to other groups (Figure 7C) suggesting stromal PTEN acts through JAGGED-1 in suppressing tumor formation.

\section{DISCUSSION}

\section{Stromal PTEN regulates mammary gland morphogenesis}

Stromal fibroblasts maintain the architecture of the mammary gland by regulating ECM deposition, secreting growth factors and facilitating direct mesenchymal-epithelial interactions. ${ }^{15,28}$ It is therefore logical to speculate that genetic alterations within fibroblasts could result in a multitude of disorders, including cancer. Recent evidence suggests that genetic manipulation of stromal cells can transform adjacent epithelium even in the absence of pre-existing hyperplasia. 1 , 29, 30 Our own work demonstrates that stromal PTEN loss induces changes in ECM deposition, immune cell infiltration and oncogenic factor secretion, thereby enabling malignant transformation of adjacent pre-neoplastic ErbB2 epithelial cells and accelerating tumor growth. ${ }^{7,}{ }^{16}$ Despite this evidence, how fibroblasts reprogram preneoplastic epithelial cells at the earliest stages of breast cancer is poorly understood. In this study, we characterize non-cell autonomous alterations within pre-neoplastic adjacent epithelia in response to stromal loss of PTEN. Specifically, these mice exhibited stunted ductal development and aberrant alveolar side-branching. In the mammary gland, excessive side branching can occur when the cellular microenvironment is disrupted. For example, overexpression of stromal regulators MMP-3 or MMP-14 induces unrestrained aberrant side-branching and consequent tumorigenesis. ${ }^{31,32}$ Our previous findings revealed that loss of stromal PTEN increases MMP9 activity ${ }^{7}$, which could in part explain the restricted ductal elongation and aberrant alveolar side-branching as described herein.

\section{Stromal PTEN regulates the mammary stem cell niche}

Bipotential MaSCs found in the basal/myoepithelial layer are important in both development and maintenance of mammary homeostasis. ${ }^{8,12,22,33}$ Cell fate decisions of stem cells are tightly regulated by extrinsic signals from the niche, which consists of adjacent stromal cells including fibroblasts, growth factors, cytokines and chemokines, and the ECM. ${ }^{19,} 34,35$ Here we report that both wild-type and ErbB2 mice with PTEN-null stroma exhibit an expansion of the $\mathrm{CD} 24^{+} \mathrm{CD} 29^{\mathrm{Hi}}$ MaSC-enriched population. The altered stem cell activity in vivo was confirmed using well-defined in vitro stem cell assays. It is important to note that we do not see an expansion of MaSC-enriched cells in our ErbB2 animals when compared to syngeneic WT FVB/N controls, which is consistent with previous reports. ${ }^{22,}{ }^{23}, 33 \mathrm{~A}$ significant increase in ErbB2-driven MaSC-enriched basal/myoepithelium occurs only in the context of a PTEN-null stroma. These data corroborate the increase in aberrant alveolar sidebranching also seen in these mice implicating stromal PTEN in the regulation of epithelial lineage differentiation through altering the MaSC-enriched pool.

Whether MaSCs from a PTEN-null stromal microenvironment directly alters oncogenic transformation is still unclear. Two independent studies have identified novel CD24+ Jag $1^{-}$ 
and $\mathrm{CD} 24^{+} \mathrm{Sca}^{+}$populations as potential tumor initiating cells in ErbB2-mediated tumorigenesis. ${ }^{36,37}$ Even though the link between these subpopulations and CD $24^{+} \mathrm{CD} 29^{\mathrm{Hi}}$ MaSC cells remains undefined, it is likely that the $\mathrm{CD}_{2} 4^{+} \mathrm{Jag} 1^{-}$and $\mathrm{CD} 24^{+} \mathrm{Sca} 1^{+}$ populations exist within $\mathrm{CD} 24^{+} \mathrm{CD} 29^{\mathrm{Hi}} \mathrm{MaSC}$-enriched pool and that loss of stromal PTEN similarly alters these tumor initiating subsets. Another possibility is that genetic ablation of fibroblast Pten disrupts paracrine signaling between altered MaSCs and adjacent luminal cells. This disruption would affect proliferation, differentiation and the tumorigenic potential of the luminal cells, which have been postulated to be the cellular precursors for the HER2/ ErbB2 luminal subtype of breast cancer. ${ }^{22,38}$ While beyond the scope of the current report, the data reported herein imply that fibroblast PTEN paracrine signaling possibly alters the cell of origin of ErbB2+ breast cancer. ${ }^{22,33,37,39}$

\section{Stromal Pten maintains JAGGED-1 and facilitates paracrine NOTCH3 mediated signaling in adjacent mammary epithelium}

NOTCH receptors, including NOTCH3, are known to be key regulators of stem cell maintenance and differentiation in normal tissue. ${ }^{25,40-46}$ In the mammary gland specifically, downregulation of NOTCH signaling results in the expansion of MaSCs in vivo, whereas constitutive activation of NOTCH signaling favors luminal cell fate at the expense of basal lineage commitment. ${ }^{24,45}$ These studies revealed that forced NOTCH signaling expands the luminal progenitor population leading to hyperplasia and the highly aggressive basal-like breast cancer. $^{24,45}$ Thus, NOTCH signaling determines alternate outcomes in different mammary cell types in a developmental stage dependent manner. Utilizing genetic mouse models, we show for the first time that mammary glands lacking stromal PTEN exhibit an increase in the MaSC-enriched pool that is attributed to decreased NOTCH signaling within this population corroborating previous findings. ${ }^{24,} 45$ Mechanistically, we have identified loss of JAGGED-1 within the PTEN-null mammary fibroblasts as a key mediator of this MaSC niche. Importantly, JAGGED/NOTCH signaling has been well-described to be oncogenic in the breast tumor microenvironment, which may seemingly contradict our findings. ${ }^{47-52}$ However, these previous experiments utilized immortalized breast cancer cell lines to discern the function of stromal JAGGED/NOTCH in promotion of pre-existing tumors. In contrast, our study is quite distinct in that we have focused on the pre-neoplastic MaSC niche to dissect the role of this pathway in normal mammary gland development. Given that low stromal PTEN in combination with low stromal JAG1 is more predictive of earlier breast cancer patient recurrence compared to low stromal expression of either PTEN or $J A G 1$ alone, future studies will focus on defining the association between stromal PTEN and JAGGED-1 in breast cancer.

Combined, we show for the first time that loss of stromal PTEN regulates the pre-neoplastic mammary stem cell niche through 'altered' JAGGED-1 to NOTCH3 paracrine signaling. Based on these results, we propose that at least one of the mechanisms by which stromal PTEN suppresses ErbB2 induced tumor growth is through blocking the expansion of the MaSC-enriched pool. These results highlight a novel role for stromal PTEN in mammary morphogenesis and provide new insights into the complex cross-talk between fibroblasts and the MaSC niche. 


\section{MATERIALS AND METHODS}

\section{Ethics Statement}

Animal use was in compliance with federal and University Laboratory Animal Resources regulations under protocols 2007A0120-R1 (MCO) and 2007A0239-R2 (GL) approved by the OSU Institutional Animal Care and Use Committee (IACUC).

\section{Transgenic Mice}

Fsp-cre mice were generated previously and confirmed to express $F_{s} p$ specifically within the mammary stromal fibroblasts, with absence in the epithelium, macrophages and endothelium. ${ }^{6,7}$ Pten $^{f l f f l}$ mice were created and maintained as reported. ${ }^{7}$ MMTV-ErbB2 mice were generously provided by Dr. William Muller. ${ }^{53}$ All mice were $8-10$ wks of age and at least tenth-generation congenic (N10) FVB/N. Wild-type FVB/N were purchased from Jackson Laboratories (Bar Harbor, Maine, USA). Primary mouse MMFs were isolated as described. ${ }^{54}$

\section{Whole-mount analysis}

Inguinal mammary glands were harvested as described. ${ }^{55}$ Mice in diestrus were excluded. Briefly, ductal length was determined by measuring from the farthest edge of the lymph node (relative to the nipple) to the longest duct. The longest three ducts were averaged per gland and the two inguinal glands averaged per mouse. Alveolar side-branching was determined by counting all aberrant ducts within a representative $4 \mathrm{x}$ field per gland and the two inguinal mammary glands were averaged per mouse.

\section{Fluorescence activated cell sorting}

Mammary tissue was harvested as described (4-8 mice/genotype were pooled/experiment). ${ }^{23}$

Briefly, tissue was dissociated with a McIlwain tissue chopper (Mickle Laboratory Engineering, Guildford, Surrey, United Kingdom) and placed in Epicult-B medium (Stem Cell Technologies, Vancouver, British Columbia, Canada) supplemented with 5\%FBS, 300U/ml Collagenase (Sigma, St. Louis, Missouri, USA) and 100U/ml hyaluronidase (Sigma), and digested for $1 \mathrm{hr}$ at $37^{\circ} \mathrm{C}$ with gentle shaking. Red blood cells were lysed in ammonium chloride (Stem Cell Technologies). The digested tissue was then sequentially dissociated in $0.25 \%$ trypsin-EDTA (Sigma) for $1 \mathrm{~min}, 5 \mathrm{mg} / \mathrm{ml}$ Dispase (Stem Cell Technologies) plus $0.1 \mathrm{mg} \mathrm{ml}^{-1}$ DNase1 (Sigma) for $1 \mathrm{~min}$ and filtered through a $40 \mu \mathrm{m}$ cell strainer. Single cell populations were subjected to a modified negative selection process to obtain lineage negative $\left(\mathrm{Lin}^{-}\right)$subpopulations. ${ }^{11}$ Specifically, CD45 ${ }^{+}$, Ter119 ${ }^{+}, \mathrm{CD} 31^{+}$and BP- $1^{+}$cells were removed using the EasySep Mammary Epithelial Cell Enrichment kit as per manufacturer's instructions (Stem Cell Technologies). Lin $^{-}$cells were further stained for CD24-PE (\#553262, BD Pharmingen, San Jose, California, USA), CD29-FITC (\#555005, BD Pharmingen) and CD61-APC (\#MCD6105; Invitrogen, Grand Island, New York, USA). ${ }^{23,33,56}$ Isotype controls for each antibody were used: PE-Rat IgG2b, $\kappa$ isotype (\#555848, BD Pharmingen); FITC-Hamster IgM, $\lambda 1$ isotype (\#553960, BD Pharmingen); APC-Hamster IgG, (\#17-488-81, eBiosciences, San Diego, California, USA). All procedures were performed using FACSAria (BD Biosciences, San Jose, California, USA). Gating 
excluded cells labeled with isoform-matched control antibodies. If isotype-control was unclean, experiment was excluded. Viable cells were determined by DAPI exclusion. For downstream assays, viable cell numbers were determined by Trypan Blue exclusion. All data analyses were performed using FlowJo single cell analysis software.

\section{In vitro stem/progenitor cell assays}

For colony forming assays, sorted MaSC-enriched or Lin-CD $24^{+} \mathrm{CD} 29^{+}$cells were seeded onto a feeder layer of mitomycin C (Roche, Indianapolis, Indiana, USA) growth-arrested NIH3T3 cells (ATCC; grown in 10\%DMEM + 1\% penicillin/streptomycin), Pten ${ }^{f l / f l}$, or Fspcre; Pten ${ }^{f l / f l}$ MMFs in 6-well plates at a density of 2,000 cells/well. Fsp-cre;Pten ${ }^{f l / f l}$ MMFs were transiently transfected with empty vector (pENT-CMV4-EF1alpha-GFP) or mouse Jag1 (mJAG1) using jetPEI® (Polyplus Transfections, NY, NY, USA), mitomycin C treated on day 2 post-transfection, and epithelia added on day 3 post-transfection. The mJAG1 construct was engineered by Welgen, Inc. PCR amplified mJAG1 (GE Dharmacon MMM1013-202859638) was inserted into pENT-CMV-EF1alpha-GFP via EcoR1 and XbaI. Pten $^{f l / f l}$ MMFs were treated with vehicle (DMSO) or GSI-XXI (EMD Millipore, Billerica,

Massachusetts, USA). After $24 \mathrm{hrs,} \mathrm{the} \mathrm{media} \mathrm{was} \mathrm{replaced} \mathrm{with} \mathrm{serum-free} \mathrm{culture} \mathrm{medium}$ as described. ${ }^{23}$ 7-10 days later, colonies were fixed in ice-cold acetone:methanol (1:1), stained with Giemsa and \% colony area assessed by a user defined threshold followed by analyzing particles with defined circularity in Image ${ }^{57}$. To assess organoid formation, isolated MaSC-enriched cells were suspended in epithelial media (Stem Cell Technologies) with 5\% FBS and then seeded within 50- $\mu$ l growth factor-reduced matrigel (BD Biosciences) in an 8-well chamber slide at a density of 20,000 cells/well. After 10 days, individual 50- $\mu$ matrigel cultures were embedded in histogel (Richard-Allan Scientific, Grand Island, New York, USA) and fixed in 4\% paraformaldehyde. These were sectioned and stained with hematoxylin and eosin (H\&E) or processed for immunostaining. For mammosphere assays, single sorted MaSC-enriched cells were plated in ultralow attachment 24-well plates (Corning, Tewksbury, Massachusetts, USA) at a density of 10,000 cells/well in serum-free mammary epithelial basal medium (Stem Cell Technologies) plus B27 (Life Technologies, Grand Island, New York, USA), 20ng/ml EGF (Sigma), 20ng/ml bFGF (PeproTech, Rocky Hill, New Jersey, USA) and 4ug/ml heparin (Sigma) as described. Mammosphere cultures were grown for 14 days and all observable mammospheres counted. ${ }^{58}$

\section{Phase contrast imaging and immunostaining}

All live cell imaging was taken on the EVOS XL Core (Life Technologies). For immunofluorescence (IF), paraffin-embedded tissue and matrigel sections were dewaxed, and subjected to antigen retrieval by steaming samples in DAKO Target retrieval solution (pH 6.1) (Carpinteria, California, USA) for at least 20 mins before blocking with the DAKO antibody diluent. The following primary antibodies were then used: CK8 (\#TROMA-1, 1:400, Developmental Studies Hybridoma Bank, Iowa City, Iowa, USA), CK5 (\#ab24647, 1:200, Abcam, Cambridge, Massachusetts, USA), a-SMA (\#A2547, 1:400, Sigma), JAGGED-1 (\#2620, 1:50, Cell Signaling, Danvers, Massachusetts, USA) and NOTCH3 (\#ab23426, 1:400, Abcam). Secondary detection was performed using antibodies conjugated to AlexaFluor dyes (Invitrogen; Molecular Probes) for $1 \mathrm{hr}$ at room temperature. Sections 
were mounted with SlowFade ${ }^{\circledR}$ Gold Antifade mount with DAPI (Life Technologies). Representative fluorescent images were taken on an Eclipse E800 microscope (Nikon Instruments Inc., Melville, New York, USA) using the MetaVue ${ }^{\mathrm{TM}}$ Research Imaging system (Molecular Devices, Sunnyvale, California, USA) and images for NOTCH3 and JAGGED-1 quantification were taken on the PerkinElmer (Hopkinton, Massachusetts, USA) Vectra Automated Quantitative Pathology Imaging system (VECTRA®).

\section{NOTCH3 and JAGGED-1 Quantification}

At least three 40x images were quantified per mammary gland. NOTCH3 in the basal/ myoepithelium was determined using Image ${ }^{57}$ by separating the channels and individually adjusting the threshold for the green (a-SMA) and red (NOTCH3) until only the myoepithelial area staining was visible. The total area of each channel was determined by analyzing particles, and the percentage of red/green calculated. Stromal JAGGED-1 images were quantified using the pattern recognition algorithm in the Inform ${ }^{\circledR}$ software (PerkinElmer). Briefly, each image underwent manual tissue segmentation to select only the stroma adjacent to the mammary epithelium for scoring. Other cell types (macrophages, adipocytes, blood vessels) were manually excluded from the stromal area to be scored. The spectrally unmixed Alexafluor-594 signal (JAGGED-1 positivity) was scored based on a user defined threshold into four categories $(0+, 1+, 2+$ and $3+)$. The percent of cells within each scoring category was determined based on cell segmentation by DAPI. An H-Score was then calculated using following formula: [1x(\%cells $1+)+2 x(\%$ cells $2+)+3 x(\%$ cells $3+)]$.

\section{Quantitative Real-time PCR and immunoblots}

Total RNA was obtained using TRIzol (Invitrogen), treated with DNAse I (DNA-free, Ambion, Grand Island, New York, USA), and cDNA produced using SuperScript III Reverse Transcriptase (Invitrogen). For cDNA generated from FACS isolated MaSC-enriched, luminal progenitor and mature luminal populations, a pre-amplification step was performed following the TaqMan ${ }^{\circledR}$ (Applied Biosystems, Grand Island, New York, USA) PreAmp Master Mix protocol as per manufacturer's instructions. qRT-PCR was performed using TaqMan® Gene Expression Assays and Roche Universal Probe Library primers (listed in Supplementary Methods). Sample quality was verified by comparing $\mathrm{C}_{\mathrm{t}}$ values for Gapdh. For immunoblotting, fibroblasts were lysed on ice [50mM Tris-HCl, pH7.4; $100 \mathrm{mM} \mathrm{NaCl}$; 1mM EDTA; 1mM EGTA; 1mM NaF; 0.1\% SDS; 0.5\% Sodium Deoxycholate; $1 \%$ TritonX-100; 10\% Glycerol; Protease and Phosphatase Inhibitor Cocktails (Sigma)], and protein levels quantified (Bradford Assay, Bio-Rad, Hercules, California, USA). Protein lysate was resolved using SDS-PAGE, and transferred to PVDF membrane (EMD Millipore, Billerica, Massachusetts, USA). The LiCOR Odyssey TBS Blocking Buffer (Lincoln, Nebraska, USA) was used to block and as a diluent for both primary (Jagged-1-\#2620, Cell Signaling; $\beta$ actin-\#A1978, Sigma) and secondary antibodies (LiCOR). Signal was detected using the LiCOR Odyssey®.

\section{Statistical Methods}

Sample size was not pre-determined statistically. All conclusions were determined by analyzing distinct genetic groups in a blinded fashion. Within each genetic group, mice were randomly utilized. For data of sample sizes >five, normal distribution was determined by 
Kolmogorov-Smirnov Goodness-of-Fit. Sample variance was determined by F-test. Statistical comparison of multiple groups was performed by ANOVA while direct comparison between two groups of normally distributed data was done by homoscedastic or heteroscedastic Student's $t$-test as appropriate. All analyses using Student's $t$-test were twotailed except confirmation by qRT-PCR. For data not normally distributed, statistical comparisons were done by Mann-Whitney. For JAG1 and PTEN related survival analyses, patients in the Finak ${ }^{27}$ dataset were separated into low (bottom quartile) and high (remaining) expressing groups. Kaplan-Meier survival curves were generated to evaluate survival over a three year period. Statistical significance was determined using log-rank.

\section{Supplementary Material}

Refer to Web version on PubMed Central for supplementary material.

\section{ACKNOWLEDGMENTS}

We thank Jason Bice and Daphne Bryant (Solid Tumor Biology Program-Histology Core), Bryan McElwain and Katrina Miller (OSUCCC-Analytic Cytometry) and Sarah Warner (OSUCCC-Genomics) for technical support. This study was supported by the NIH (PO1CA097189, M.C.O. and G.L.), the Department of Defense (W81XWH-14-1-0040, G.M.S.) and the Pelotonia Fellowship Program (S.B. and G.M.S). Any opinions, findings, and conclusions expressed in this material are those of the author(s) and do not necessarily reflect those of the Pelotonia Fellowship Program.

\section{REFERENCES}

1. Bhowmick NA, Neilson EG, Moses HL. Stromal fibroblasts in cancer initiation and progression. Nature. 2004; 432:332-337. [PubMed: 15549095]

2. Parsonage G, Filer AD, Haworth O, Nash GB, Rainger GE, Salmon M, et al. A stromal address code defined by fibroblasts. Trends in immunology. 2005; 26:150-156. [PubMed: 15745857]

3. Tomasek JJ, Gabbiani G, Hinz B, Chaponnier C, Brown RA. Myofibroblasts and mechanoregulation of connective tissue remodelling. Nature reviews Molecular cell biology. 2002; 3:349363. [PubMed: 11988769]

4. Kalluri R, Zeisberg M. Fibroblasts in cancer. Nature reviews Cancer. 2006; 6:392-401. [PubMed: 16572188]

5. Mueller MM, Fusenig NE. Friends or foes - bipolar effects of the tumour stroma in cancer. Nature reviews Cancer. 2004; 4:839-849. [PubMed: 15516957]

6. Trimboli AJ, Fukino K, de Bruin A, Wei G, Shen L, Tanner SM, et al. Direct evidence for epithelialmesenchymal transitions in breast cancer. Cancer research. 2008; 68:937-945. [PubMed: 18245497]

7. Trimboli AJ, Cantemir-Stone CZ, Li F, Wallace JA, Merchant A, Creasap N, et al. Pten in stromal fibroblasts suppresses mammary epithelial tumours. Nature. 2009; 461:1084-1091. [PubMed: 19847259]

8. Bocker W, Moll R, Poremba C, Holland R, Van Diest PJ, Dervan P, et al. Common adult stem cells in the human breast give rise to glandular and myoepithelial cell lineages: a new cell biological concept. Laboratory investigation; a journal of technical methods and pathology. 2002; 82:737-746. [PubMed: 12065684]

9. Dontu G, Al-Hajj M, Abdallah WM, Clarke MF, Wicha MS. Stem cells in normal breast development and breast cancer. Cell proliferation. 2003; 1(36 Suppl):59-72.

10. Clarke RB, Spence K, Anderson E, Howell A, Okano H, Potten CS. A putative human breast stem cell population is enriched for steroid receptor-positive cells. Developmental biology. 2005; 277:443-456. [PubMed: 15617686]

11. Stingl J, Eirew P, Ricketson I, Shackleton M, Vaillant F, Choi D, et al. Purification and unique properties of mammary epithelial stem cells. Nature. 2006; 439:993-997. [PubMed: 16395311] 
12. Vaillant F, Asselin-Labat ML, Shackleton M, Lindeman GJ, Visvader JE. The emerging picture of the mouse mammary stem cell. Stem cell reviews. 2007; 3:114-123. [PubMed: 17873344]

13. Rios AC, Fu NY, Lindeman GJ, Visvader JE. In situ identification of bipotent stem cells in the mammary gland. Nature. 2014; 506:322-327. [PubMed: 24463516]

14. Richert MM, Schwertfeger KL, Ryder JW, Anderson SM. An atlas of mouse mammary gland development. Journal of mammary gland biology and neoplasia. 2000; 5:227-241. [PubMed: 11149575]

15. Wiseman BS, Werb Z. Stromal effects on mammary gland development and breast cancer. Science. 2002; 296:1046-1049. [PubMed: 12004111]

16. Bronisz A, Godlewski J, Wallace JA, Merchant AS, Nowicki MO, Mathsyaraja H, et al. Reprogramming of the tumour microenvironment by stromal PTEN-regulated miR-320. Nature cell biology. 2012; 14:159-167.

17. Boquest AC, Shahdadfar A, Fronsdal K, Sigurjonsson O, Tunheim SH, Collas P, et al. Isolation and transcription profiling of purified uncultured human stromal stem cells: alteration of gene expression after in vitro cell culture. Molecular biology of the cell. 2005; 16:1131-1141. [PubMed: 15635089]

18. Lim E, Vaillant F, Wu D, Forrest NC, Pal B, Hart AH, et al. Aberrant luminal progenitors as the candidate target population for basal tumor development in BRCA1 mutation carriers. Nature medicine. 2009; 15:907-913.

19. Kessenbrock K, Wang CY, Werb Z. Matrix metalloproteinases in stem cell regulation and cancer. Matrix biology : journal of the International Society for Matrix Biology. 2015; 44-46:184-190. [PubMed: 25661772]

20. Plaks V, Kong N, Werb Z. The cancer stem cell niche: how essential is the niche in regulating stemness of tumor cells? Cell stem cell. 2015; 16:225-238. [PubMed: 25748930]

21. Polyak K, Kalluri R. The role of the microenvironment in mammary gland development and cancer. Cold Spring Harbor perspectives in biology. 2010; 2:a003244. [PubMed: 20591988]

22. Visvader JE. Keeping abreast of the mammary epithelial hierarchy and breast tumorigenesis. Genes \& development. 2009; 23:2563-2577. [PubMed: 19933147]

23. Shackleton M, Vaillant F, Simpson KJ, Stingl J, Smyth GK, Asselin-Labat ML, et al. Generation of a functional mammary gland from a single stem cell. Nature. 2006; 439:84-88. [PubMed: 16397499]

24. Bouras T, Pal B, Vaillant F, Harburg G, Asselin-Labat ML, Oakes SR, et al. Notch signaling regulates mammary stem cell function and luminal cell-fate commitment. Cell stem cell. 2008; 3:429-441. [PubMed: 18940734]

25. Chiba S. Notch signaling in stem cell systems. Stem cells. 2006; 24:2437-2447. [PubMed: 16888285]

26. Lobry C, Oh P, Mansour MR, Look AT, Aifantis I. Notch signaling: switching an oncogene to a tumor suppressor. Blood. 2014; 123:2451-2459. [PubMed: 24608975]

27. Finak G, Bertos N, Pepin F, Sadekova S, Souleimanova M, Zhao H, et al. Stromal gene expression predicts clinical outcome in breast cancer. Nature medicine. 2008; 14:518-527.

28. Bissell MJ, Rizki A, Mian IS. Tissue architecture: the ultimate regulator of breast epithelial function. Current opinion in cell biology. 2003; 15:753-762. [PubMed: 14644202]

29. Radisky DC, Bissell MJ. Cancer. Respect thy neighbor! Science. 2004; 303:775-777. [PubMed: 14764858]

30. Tlsty TD, Hein PW. Know thy neighbor: stromal cells can contribute oncogenic signals. Current opinion in genetics \& development. 2001; 11:54-59. [PubMed: 11163151]

31. Ha HY, Moon HB, Nam MS, Lee JW, Ryoo ZY, Lee TH, et al. Overexpression of membrane-type matrix metalloproteinase-1 gene induces mammary gland abnormalities and adenocarcinoma in transgenic mice. Cancer research. 2001; 61:984-990. [PubMed: 11221894]

32. Sternlicht MD, Lochter A, Sympson CJ, Huey B, Rougier JP, Gray JW, et al. The stromal proteinase MMP3/stromelysin-1 promotes mammary carcinogenesis. Cell. 1999; 98:137-146. [PubMed: 10428026] 
33. Vaillant F, Asselin-Labat ML, Shackleton M, Forrest NC, Lindeman GJ, Visvader JE. The mammary progenitor marker CD61/beta3 integrin identifies cancer stem cells in mouse models of mammary tumorigenesis. Cancer research. 2008; 68:7711-7717. [PubMed: 18829523]

34. Fuchs E, Tumbar T, Guasch G. Socializing with the neighbors: stem cells and their niche. Cell. 2004; 116:769-778. [PubMed: 15035980]

35. Ohlstein B, Kai T, Decotto E, Spradling A. The stem cell niche: theme and variations. Current opinion in cell biology. 2004; 16:693-699. [PubMed: 15530783]

36. Jeselsohn R, Brown NE, Arendt L, Klebba I, Hu MG, Kuperwasser C, et al. Cyclin D1 kinase activity is required for the self-renewal of mammary stem and progenitor cells that are targets of MMTV-ErbB2 tumorigenesis. Cancer cell. 2010; 17:65-76. [PubMed: 20129248]

37. Liu JC, Voisin V, Bader GD, Deng T, Pusztai L, Symmans WF, et al. Seventeen-gene signature from enriched Her2/Neu mammary tumor-initiating cells predicts clinical outcome for human HER2+:ERalpha- breast cancer. Proceedings of the National Academy of Sciences of the United States of America. 2012; 109:5832-5837. [PubMed: 22460789]

38. Forster N, Saladi SV, van Bragt M, Sfondouris ME, Jones FE, Li Z, et al. Basal cell signaling by p63 controls luminal progenitor function and lactation via NRG1. Developmental cell. 2014; 28:147-160. [PubMed: 24412575]

39. Liu JC, Deng T, Lehal RS, Kim J, Zacksenhaus E. Identification of tumorsphere- and tumorinitiating cells in HER2/Neu-induced mammary tumors. Cancer research. 2007; 67:8671-8681. [PubMed: 17875707]

40. Artavanis-Tsakonas S, Rand MD, Lake RJ. Notch signaling: cell fate control and signal integration in development. Science. 1999; 284:770-776. [PubMed: 10221902]

41. Callahan R, Egan SE. Notch signaling in mammary development and oncogenesis. Journal of mammary gland biology and neoplasia. 2004; 9:145-163. [PubMed: 15300010]

42. Dontu G, Jackson KW, McNicholas E, Kawamura MJ, Abdallah WM, Wicha MS. Role of Notch signaling in cell-fate determination of human mammary stem/progenitor cells. Breast cancer research : BCR. 2004; 6:R605-615. [PubMed: 15535842]

43. Reedijk M, Odorcic S, Chang L, Zhang H, Miller N, McCready DR, et al. High-level coexpression of JAG1 and NOTCH1 is observed in human breast cancer and is associated with poor overall survival. Cancer research. 2005; 65:8530-8537. [PubMed: 16166334]

44. Robinson GW. Using notches to track mammary epithelial cell homeostasis. Cell stem cell. 2008; 3:359-360. [PubMed: 18940725]

45. Xu K, Usary J, Kousis PC, Prat A, Wang DY, Adams JR, et al. Lunatic fringe deficiency cooperates with the Met/Caveolin gene amplicon to induce basal-like breast cancer. Cancer cell. 2012; 21:626-641. [PubMed: 22624713]

46. Patel PH, Dutta D, Edgar BA. Niche appropriation by Drosophila intestinal stem cell tumours. Nature cell biology. 2015; 17:1182-1192. [PubMed: 26237646]

47. Ghiabi P, Jiang J, Pasquier J, Maleki M, Abu-Kaoud N, Halabi N, et al. Breast cancer cells promote a notch-dependent mesenchymal phenotype in endothelial cells participating to a pro-tumoral niche. Journal of translational medicine. 2015; 13:27. [PubMed: 25623554]

48. Ghiabi P, Jiang J, Pasquier J, Maleki M, Abu-Kaoud N, Rafii S, et al. Endothelial cells provide a notch-dependent pro-tumoral niche for enhancing breast cancer survival, stemness and prometastatic properties. PloS one. 2014; 9:e112424. [PubMed: 25380486]

49. Qiu M, Peng Q, Jiang I, Carroll C, Han G, Rymer I, et al. Specific inhibition of Notch1 signaling enhances the antitumor efficacy of chemotherapy in triple negative breast cancer through reduction of cancer stem cells. Cancer letters. 2013; 328:261-270. [PubMed: 23041621]

50. Xing F, Kobayashi A, Okuda H, Watabe M, Pai SK, Pandey PR, et al. Reactive astrocytes promote the metastatic growth of breast cancer stem-like cells by activating Notch signalling in brain. EMBO molecular medicine. 2013; 5:384-396. [PubMed: 23495140]

51. Xing F, Okuda H, Watabe M, Kobayashi A, Pai SK, Liu W, et al. Hypoxia-induced Jagged2 promotes breast cancer metastasis and self-renewal of cancer stem-like cells. Oncogene. 2011; 30:4075-4086. [PubMed: 21499308] 
52. Yamamoto M, Taguchi Y, Ito-Kureha T, Semba K, Yamaguchi N, Inoue J. NF-kappaB non-cellautonomously regulates cancer stem cell populations in the basal-like breast cancer subtype. Nature communications. 2013; 4:2299.

53. Muller WJ, Sinn E, Pattengale PK, Wallace R, Leder P. Single-step induction of mammary adenocarcinoma in transgenic mice bearing the activated c-neu oncogene. Cell. 1988; 54:105-115. [PubMed: 2898299]

54. Soule HD, McGrath CM. A simplified method for passage and long-term growth of human mammary epithelial cells. In vitro cellular \& developmental biology : journal of the Tissue Culture Association. 1986; 22:6-12. [PubMed: 2418007]

55. Bernardo GM, Lozada KL, Miedler JD, Harburg G, Hewitt SC, Mosley JD, et al. FOXA1 is an essential determinant of ERalpha expression and mammary ductal morphogenesis. Development. 2010; 137:2045-2054. [PubMed: 20501593]

56. Lo PK, Kanojia D, Liu X, Singh UP, Berger FG, Wang Q, et al. CD49f and CD61 identify Her2/ neu-induced mammary tumor-initiating cells that are potentially derived from luminal progenitors and maintained by the integrin-TGFbeta signaling. Oncogene. 2012; 31:2614-2626. [PubMed: 21996747]

57. Abramoff MD, Magelhaes PJ, Ram SJ. Image Processing with ImageJ. Biophotonics International. 2004; 11:36-42.

58. Dontu G, Abdallah WM, Foley JM, Jackson KW, Clarke MF, Kawamura MJ, et al. In vitro propagation and transcriptional profiling of human mammary stem/progenitor cells. Genes \& development. 2003; 17:1253-1270. [PubMed: 12756227] 
A

B

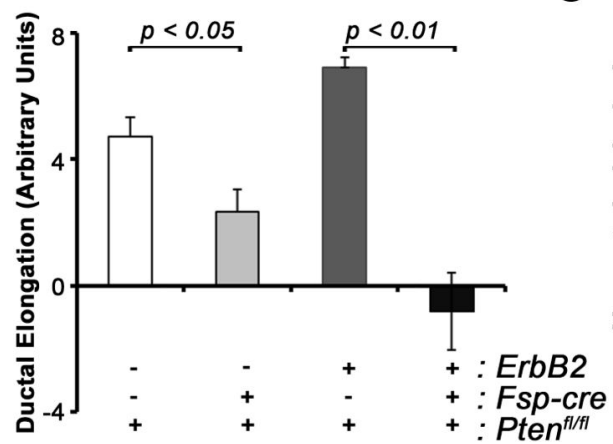

Carmine
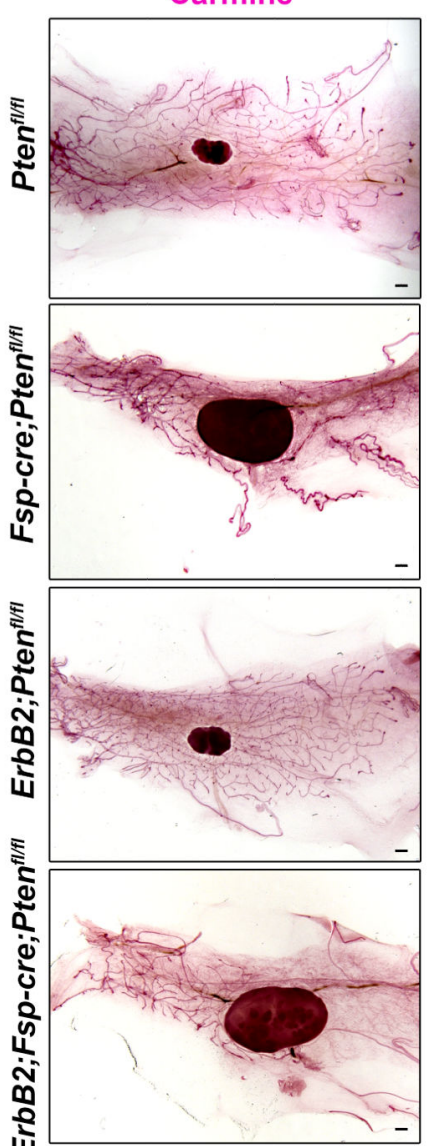

C
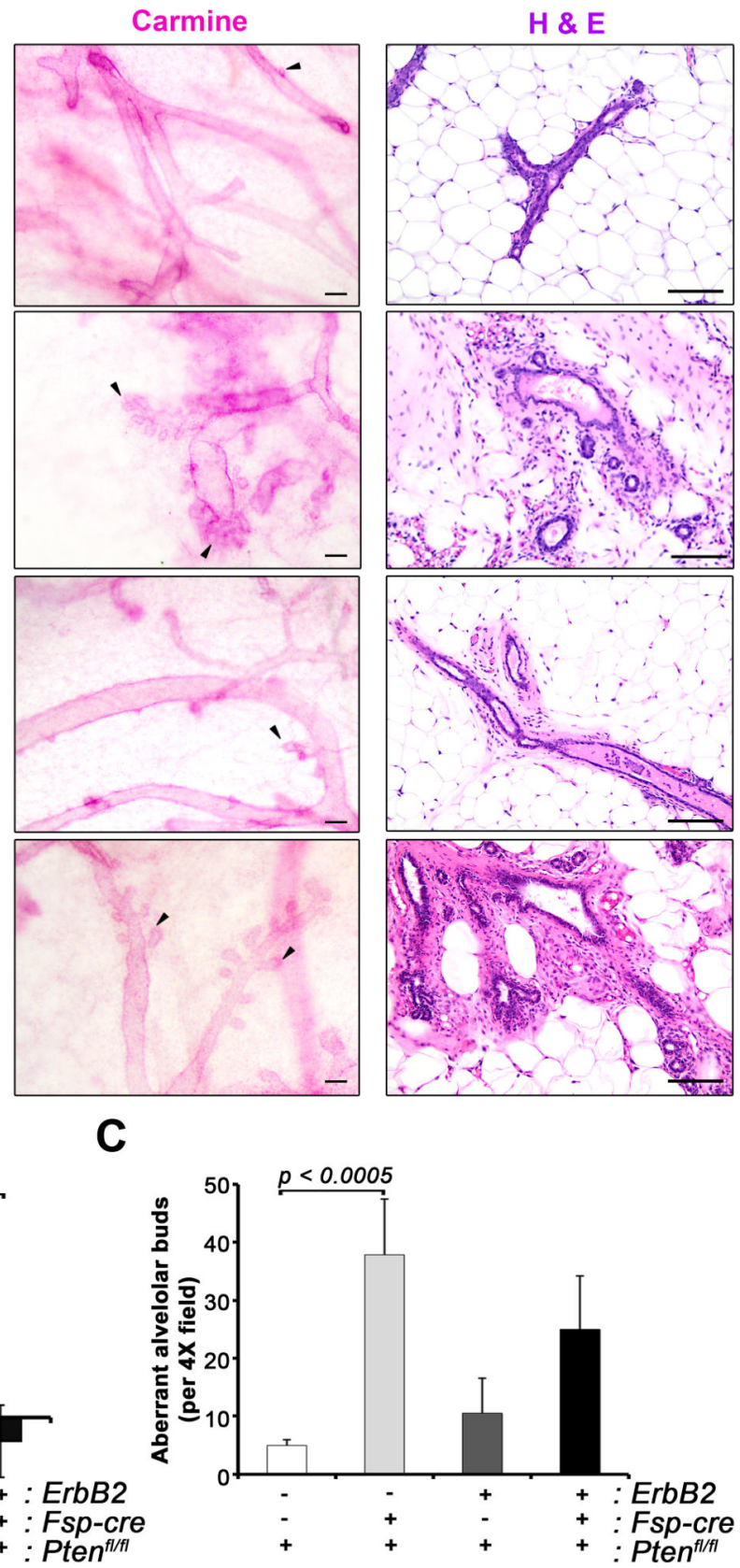

Figure 1. Loss of stromal PTEN impairs ductal elongation and promotes aberrant alveolar sidebranching

(A) Left: Representative whole mounts of mammary glands from $8 \mathrm{wk}$ old virgin mice stained with carmine alum $(\mathrm{n}=3$ per genotype; scale bars $=2 \mathrm{~mm})$. Center: Representative magnified regions to show ductal side-branching as indicated by arrowheads $(n=3$ per genotype except for ErbB2;Pten ${ }^{f l / f 1}$, n=2; scale bars=0.2mm). Right: Representative H\&E images at 8-9wks ( $\mathrm{n}=3$ per genotype; scale bars=0.1 mm). (B) Quantification of ductal length represented in A. Bars represent the mean of 3 animals per genotype plus s.e.m.; Pten ${ }^{f l / f 1} \mathrm{v}$. Fsp-cre;Pten ${ }^{f l / f 1}=\mathrm{p}<0.05 ;$ ErbB2;Pten ${ }^{f l / f 1}$ v. ErbB2:Fsp-cre;Pten ${ }^{f l / f 1}=\mathrm{p}<0.05$. Statistical analysis was performed using ANOVA followed by Student's $t$-test. (C) Quantification of 
aberrant alveolar side-branching represented in A. Bars represent the mean of 3 animals per genotype plus s.e.m. except for ErbB2;Pten ${ }^{f l / f 1}$, where the bar represents the mean of 2 animals; Pten ${ }^{f l / f l}$ v. Fsp-cre;Pten ${ }^{f l / f l}=\mathrm{p}<0.05$. Statistical analysis was performed using Student's $t$-test. 

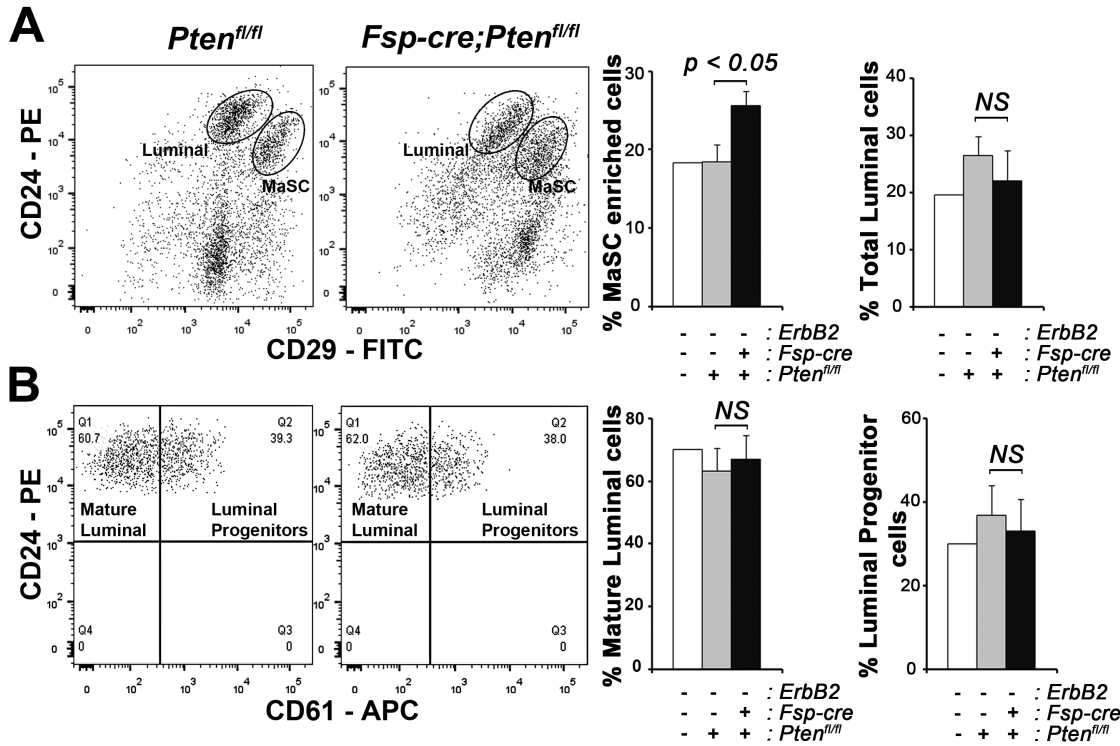

\section{ErbB2;Pten ${ }^{f / f t}$ ErbB2;Fsp-cre;Pten ${ }^{f / f t}$}
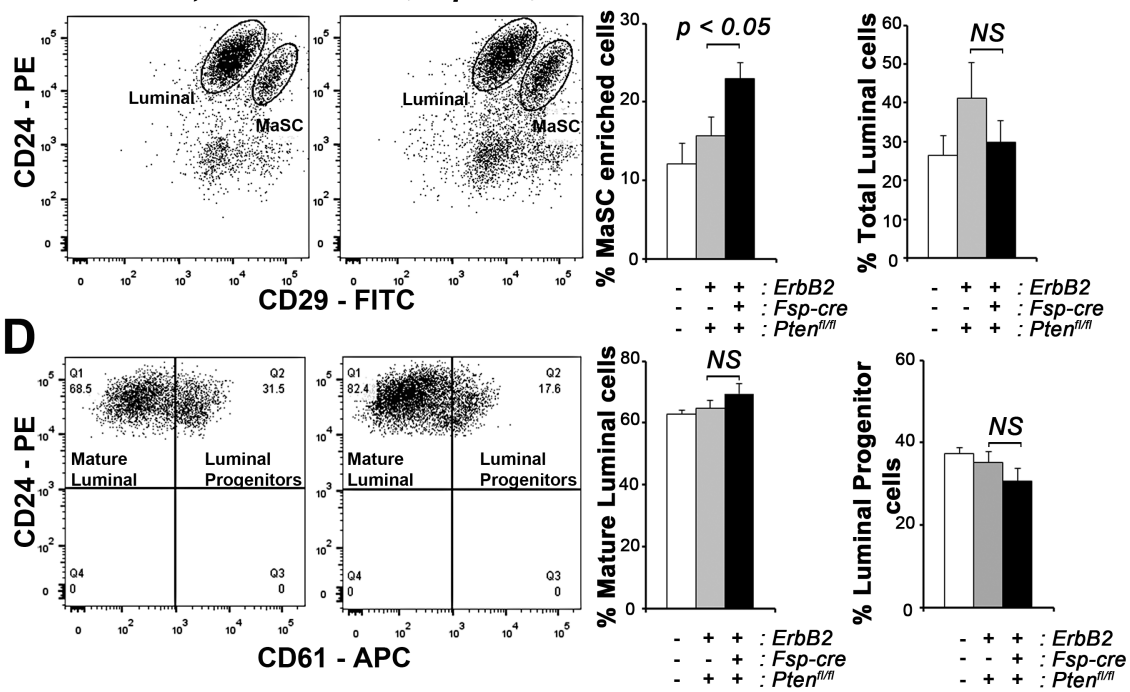

Figure 2. Loss of stromal PTEN expands the MaSC-enriched population in vivo

(A,C) Left: Representative FACS plots showing MaSC-enriched basal/myoepithelial and total luminal populations in 8-9wk old (A) Pten ${ }^{f l f l}$ v. Fsp-cre;Pten ${ }^{f l f l}$ and (C) ErbB2;Pten ${ }^{f l / f l}$ v. ErbB2;Fsp-cre;Pten ${ }^{f l / f l}$ animals. Right: Quantification of MaSC-enriched and total luminal populations represented on the left. MaSC comparison: Pten ${ }^{f l / f l}$ v. Fsp-cre;Pten ${ }^{f l / f l}=\mathrm{p}<0.05$; ErbB2;Pten ${ }^{f l / f l}$ v. ErbB2;Fsp-cre; Pten ${ }^{f l / f l}=\mathrm{p}<0.05$. (B,D) Left: Corresponding FACS plots for luminal subpopulations from $\mathrm{A}$ and $\mathrm{C}$ showing mature luminal and luminal progenitor subpopulations in 8-9wk old (B) Pten ${ }^{f l / f l}$ v. Fsp-cre;Pten ${ }^{f l / f l}$ and (D) ErbB2;Pten ${ }^{f l / f l} \mathrm{v}$. ErbB2;Fsp-cre;Pten ${ }^{f l / f l}$ animals. Right: Quantification of luminal subpopulations populations represented on the left. (A,B) Bars represent the mean of 4 independent experiments plus s.e.m, except for white bar (FVB/N control) where one experiment was performed. (C,D) Bars represent the mean of 5 independent experiments plus s.e.m for all indicated genotypes. 
Statistical analysis for A-D was carried out using ANOVA followed by Student's $t$-test (NS = non-significant). 

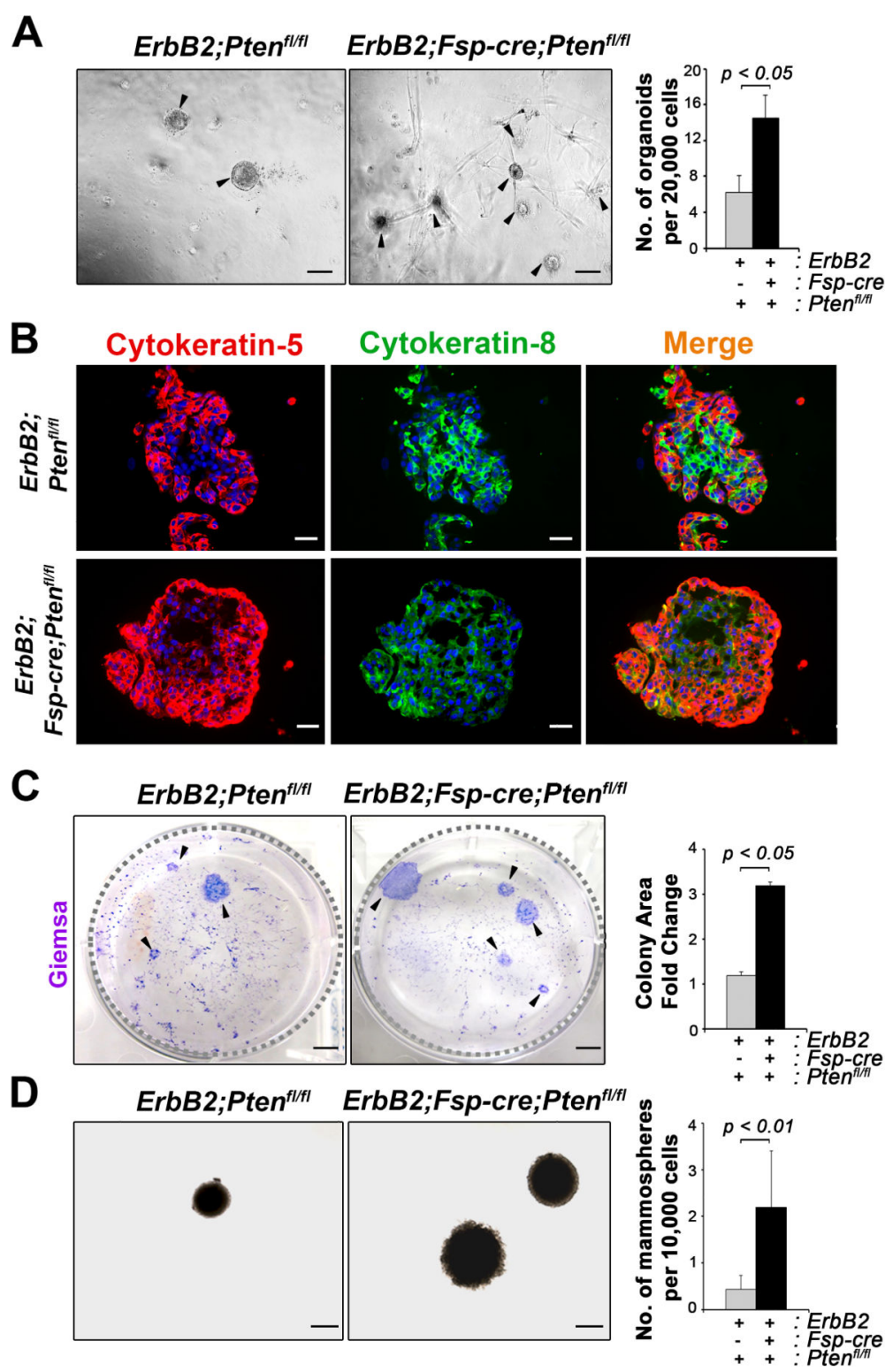

Figure 3. MaSC-enriched populations from stromal PTEN-null mice exhibit enhanced stem cell activity in vitro

(A) Left: Representative phase contrast images of MaSC-enriched derived organoids (indicated by arrowheads) in 3D matrigel. Scale bars $=0.5 \mathrm{~mm}$. Right: Quantification of organoids shown on the left. Bars represent the mean of 3 independent sorting experiments plus s.e.m. Statistical analysis was performed using Student's $t$-test ( $\mathrm{p}<0.05)$. (B) Paraffin embedded sections from MaSC-enriched derived organoids in A co-stained for CK5 and CK8 by immunofluorescence. Scale bars $=40 \mu \mathrm{m}$. (C) Left: Representative images of Giemsa stained epithelial colonies (arrowheads) on growth arrested NIH3T3 cells. Scale bars $=5 \mathrm{~mm}$. Right: Quantification depicting colony formation as represented on the left . Bars represent fold change of the total colony area from 2 independent experiments plus s.e.m. Statistical analysis was performed using Student's $t$-test ( $<<0.05)$. (D) Left:

Representative images of non-adherent mammospheres in vitro. Scale bars $=1 \mathrm{~mm}$. Right: 
Quantification of the number of mammospheres. Bars represent the mean of 3 independent sorting experiments plus s.e.m. Statistical analysis was performed using Mann-Whitney $(\mathrm{p}<0.01)$. 

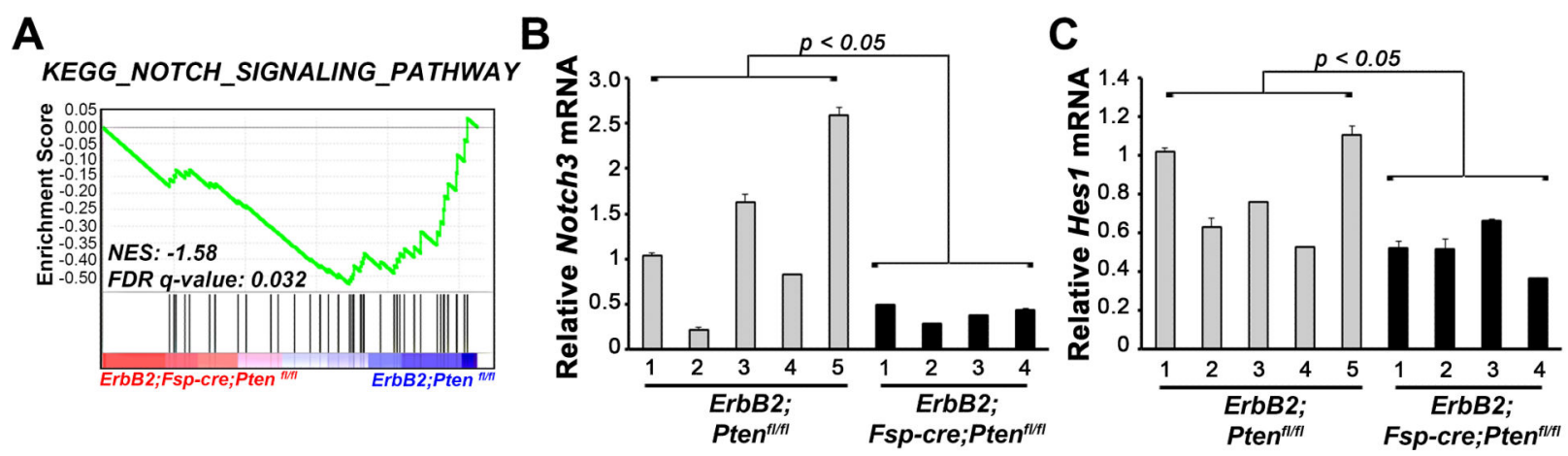

D
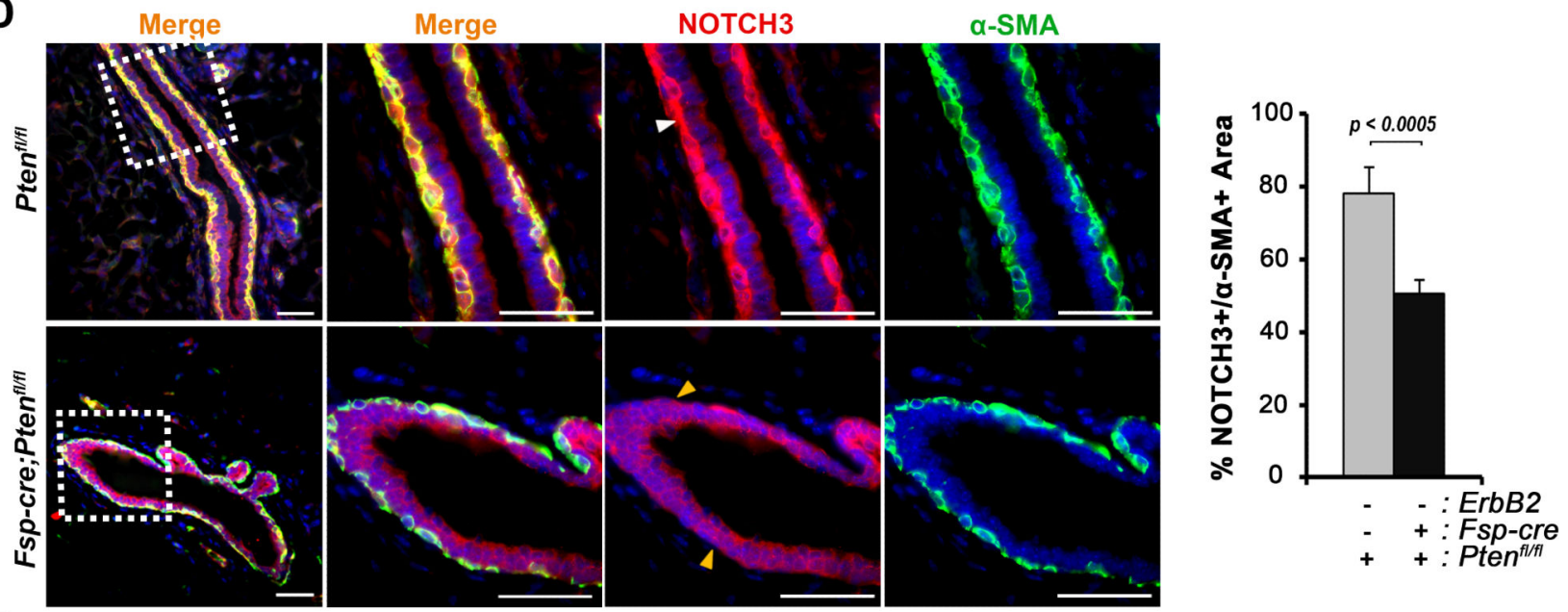

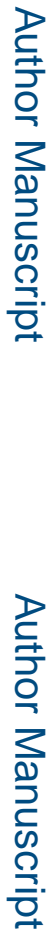
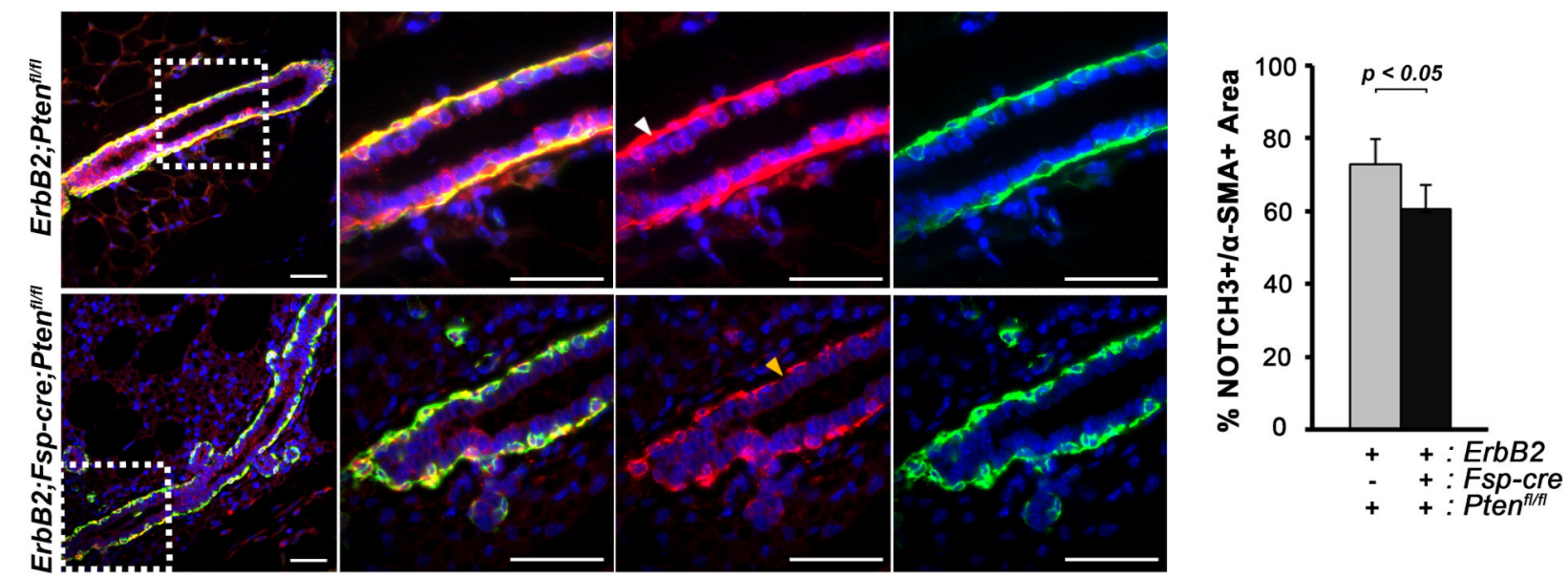

Figure 4. Stromal PTEN loss decreases NOTCH3 expression in associated MaSC basal/ myoepithelial cells

(A) GSEA depicting significant de-enrichment of NOTCH signaling pathway genes (KEGG_NOTCH_SIGNALING_PATHWAY) in MaSC-enriched cells pooled and isolated from ErbB2;Fsp-cre;Pten ${ }^{f l f l}$ mice when compared to cells pooled and isolated from ErbB2;Pten ${ }^{f l / f l}$ mice. NES = normalized enrichment score. FDR $=$ false discovery rate. $(\mathrm{B}, \mathrm{C})$ Notch3 and Hes1 mRNA expression in MaSC-enriched cells: ErbB2;Pten ${ }^{\text {fl/fl }}$ (5 independent experiments of pooled mice) v. ErbB2;Fspcre; Pten ${ }^{f l / f l}$ (4 independent experiments of pooled mice $)=p<0.05$. Bars represent mean expression of technical replicates relative to Gapdh 
plus s.e.m. Statistical analysis was performed using Student's $t$-test. (D,E) Left: Representative images depicting co-immunofluorescence of (D) Pten ${ }^{f l / f l}$ v. Fsp cre;Pten ${ }^{\text {fl/fl }}$ and (E) ErbB2;Pten ${ }^{\text {flffl }}$ v. ErbB2;Fsp-cre;Pten ${ }^{f l f l}$ mammary glands from 8-9wk old animals for NOTCH3 (red) and a-SMA (green). Scale bars $=40 \mu \mathrm{m}$. White and orange arrowheads indicate positive and negative basal/myoepithelial NOTCH3 immunofluorescence, respectively. Right: Quantification representing the mean percentage of $\mathrm{NOTCH} 3+$ area with respect to a-SMA+ area for at least 3 animals per genotype plus s.e.m: $P t e n^{f l / f l}$ v. Fsp-

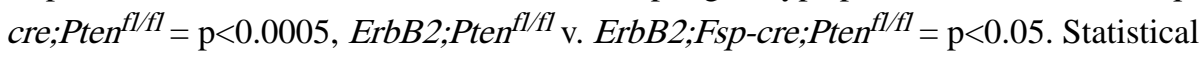
analysis was performed using Student's $t$-test. 
A

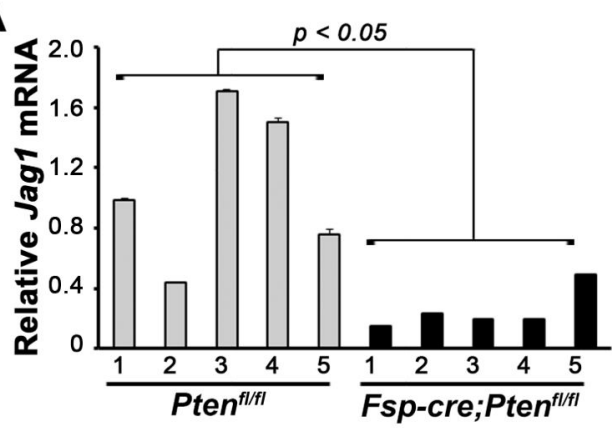

D
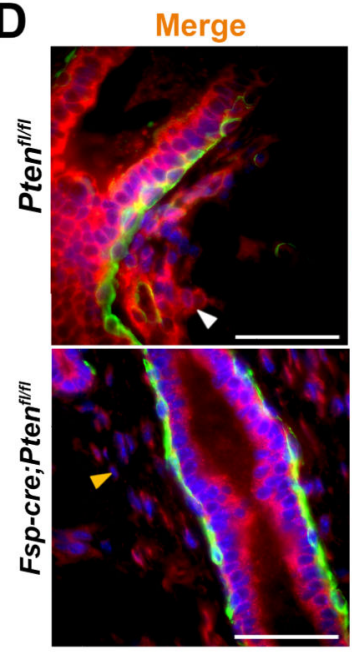

$\mathbf{E}$

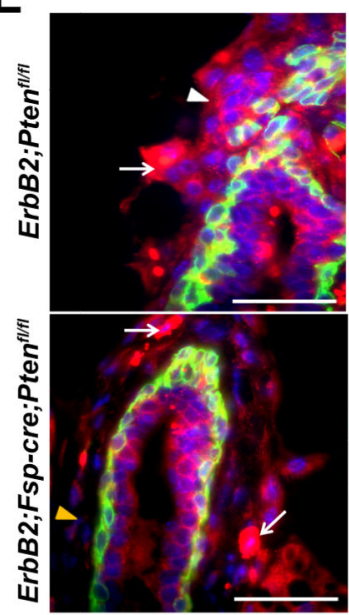

JAGGED-1
B
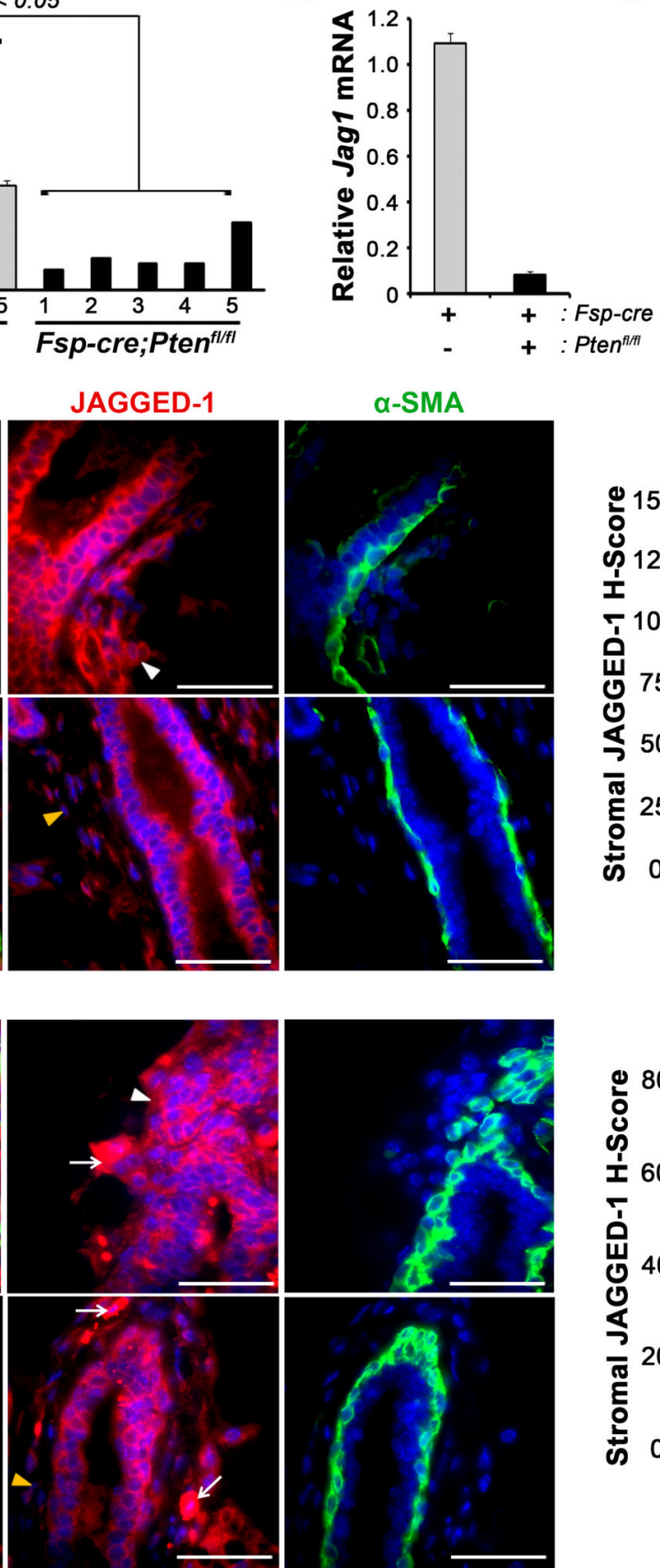

$\alpha-S M A$
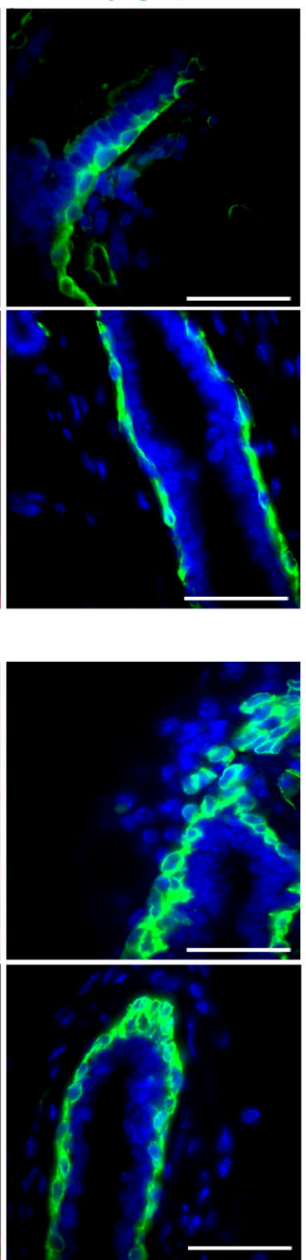

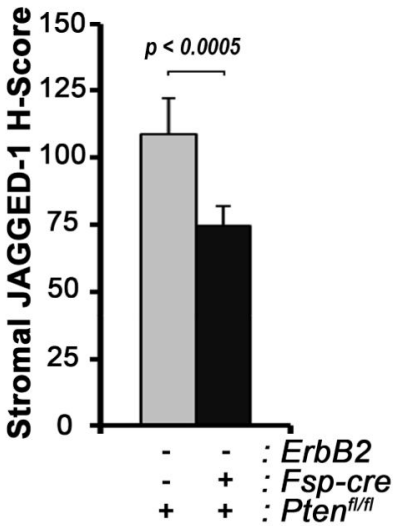

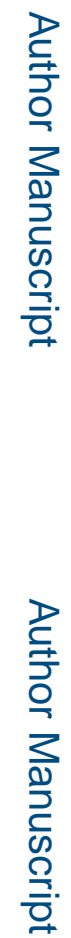

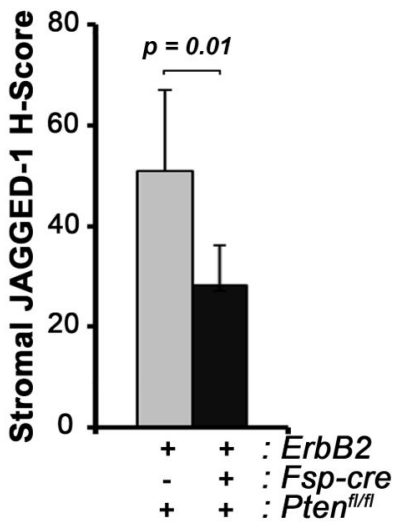

Figure 5. Loss of fibroblast PTEN decreases stromal JAGGED-1 expression (A) Jag1 mRNA in isolated primary MMFs: Pten ${ }^{f l / f l}$ v. Fsp-cre;Pten ${ }^{f l / f l}=\mathrm{p}<0.05$ (n=5 mice per genotype). Bars represent mean expression of technical replicates relative to Gapdh plus s.e.m. Statistical analysis was performed using Student's $t$-test. (B) Jag1 mRNA in immortalized Pten ${ }^{f l / f l}$ and Fsp-cre;Pten ${ }^{f l / f l}$ MMFs. Bars represent mean expression of technical replicates relative to Gapdh plus s.e.m. (C) Western blot for JAGGED-1 in the immortalized Pten ${ }^{f l / f l}$ and Fsp-cre;Pten ${ }^{f l / f l}$ MMFs. (D,E) Left: Representative images depicting co-immunofluorescence of mammary glands from (D) Pten ${ }^{f l / f l}$ v. Fsp-cre;Pten ${ }^{f l / f l}$ 
and (E) ErbB2;Pten ${ }^{f l / f l}$ v. ErbB2;Fsp-cre;Pten ${ }^{\text {fl/fl }}$ 8-9wk old animals for JAGGED-1 (red) and $a$-SMA (green). Scale bars $=40 \mu \mathrm{m}$. White and orange arrowheads indicate positive and negative stromal JAGGED-1 immunofluorescence, respectively. Arrows represent JAGGED-1 positive macrophages. Right: Bars represent the mean stromal JAGGED-1 HScore for at least 3 animals per genotype plus s.e.m: Pten $^{f l f l}$ v. Fsp-cre;Pten ${ }^{f l / f l}=\mathrm{p}<0.0005$ and ErbB2;Pten ${ }^{f l / f l}$ v. ErbB2;Fsp-cre;Pten ${ }^{f l / f l}=\mathrm{p}=0.01$. Statistical analysis was performed using (D) Student's $t$-test and (E) Mann-Whitney on independent H-Scores. 


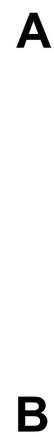

A

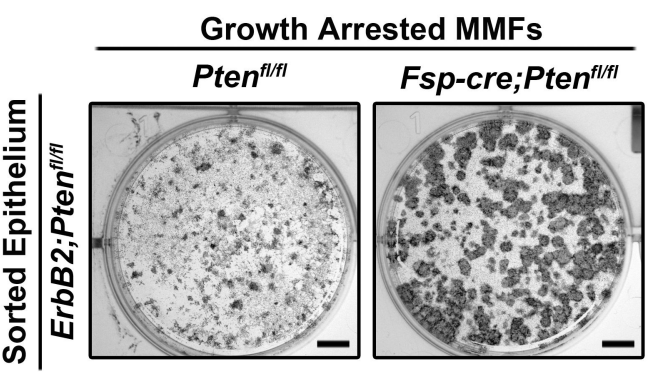

B

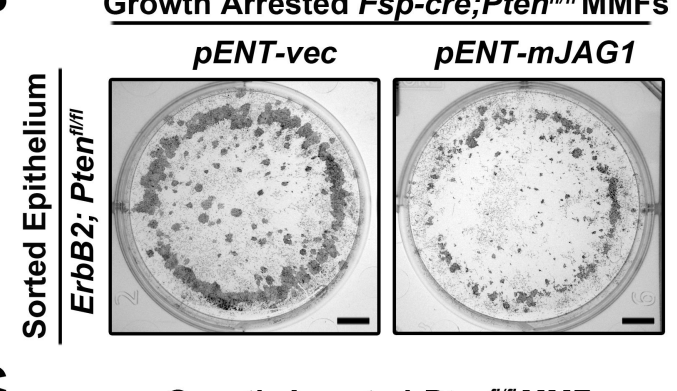

C

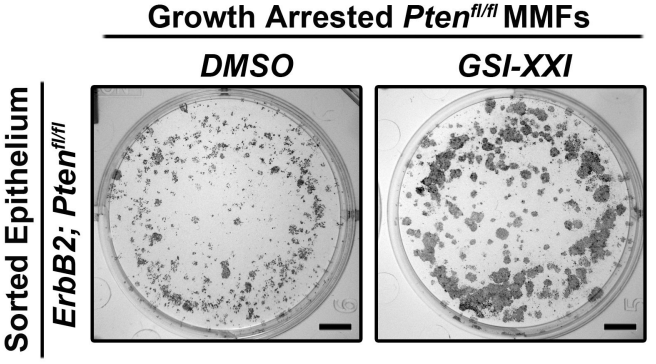

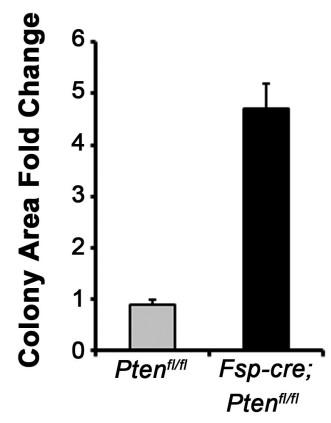
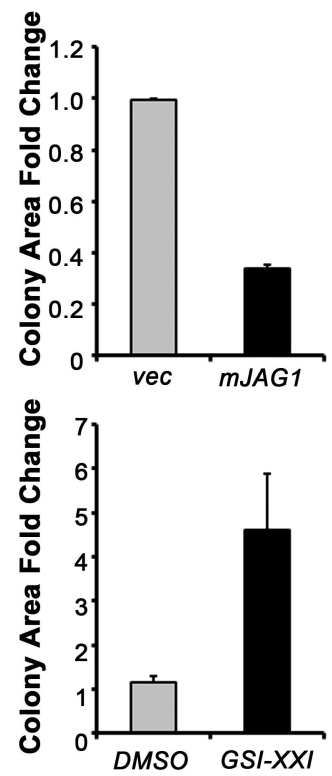

Figure 6. PTEN-null fibroblasts promote epithelial stem cell activity through JAGGED-1 (A-C) Left: Representative panels depicting Giemsa stained colonies generated by FACS isolated Lin-CD24+CD29+ ErbB2 epithelia grown on growth arrested MMFs. (A) Epithelia on Pten ${ }^{f l / f l}$ v. Fspcre;Pten ${ }^{f l / f l}$ MMFs (n=2 independent experiments using pooled epithelia from 3-4 mice; bars represent fold change of the total colony area plus s.e.m.; $\mathrm{p}<0.0001$ using Student's $t$-test on biological replicates). (B) Epithelia on $F s p$-cre;Pten ${ }^{f l / f l}$ control (pENT-vec) v. JAGGED-1 overexpressing (pENT-mJAG1) MMFs ( $\mathrm{n}=1$ experiment of 4 mice; bars represent fold change of the total colony area plus s.e.m.). (C) Epithelia on $P t e n^{f l / f l}$ MMFs treated with DMSO v. GSI-XXI ( $\mathrm{n}=2$ independent experiments of 4 mice; bars represent fold change of the total colony area plus s.e.m.; $p<0.001$ using Student's $t$-test on biological replicates). Scale bars $=5 \mathrm{~mm}$. 

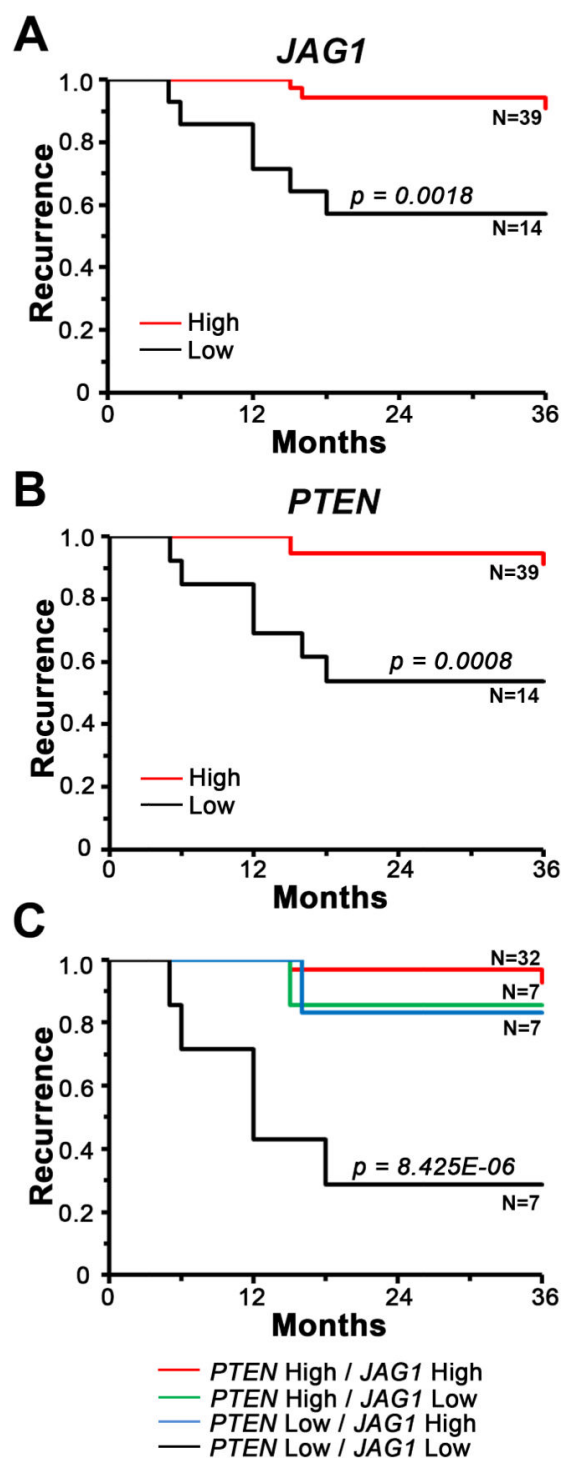

Figure 7. Low stromal $P T E N$ and low stromal $J A G 1$ correlate with shorter time to recurrence in human breast cancer patients

(A-C) Kaplan-Meier analysis exhibiting recurrence probability for the Finak patient population stratified by low (bottom quartile) v. high (remaining) (A) $J A G 1$ (low, n=14; high, n=39), (B) PTEN (low, n=14; high, n=39), and (C) combined PTEN and JAG1 (PTEN low/JAG1 low, n=7; PTENlow/JAG1 high, n=7; PTENhigh/JAG1 low, n=7; PTENhigh/ $J A G 1$ high, $\mathrm{n}=32$ ) expression. Significance determined by log-rank. 